\title{
Conexión intrínseca entre la pragmática y la gramática
}

\author{
JEREMY W. BACHELOR \\ Heartland Community College - Illinois, EE.UU.
}

Recibido el 25-05-2015; primera evaluación el 14-08-2015; segunda evaluación el 31-08-2015; aceptado el 02-09-2015

\section{Resumen}

Estudios sugieren que la gramática es un prerrequisito a la adquisición pragmática en la Lengua 2 o segunda lengua/lengua extranjera (L2), aunque una alta competencia gramatical no garantice una alta competencia pragmática. Este estudio examina extensamente la correlación entre estas dos competencias. En el año 2013, en una universidad norteamericana, 29 estudiantes de español de nivel intermedio-alto hicieron una prueba de gramática y una de pragmática. La segunda evaluó las solicitudes, las invitaciones, los rechazos y las disculpas en español. Al comparar las dos competencias, los resultados sugieren que existe una relación positiva entre la pragmática y la gramática. Por tanto, esta investigación apoya estudios previos que demuestran que la competencia gramatical de estudiantes de L2 es necesaria para el desarrollo de la competencia pragmática; sin embargo, no es suficiente para ella.

Palabras clave: pragmática, gramática, español como lengua extranjera, sociolingüística, educación

\footnotetext{
* El profesor Jeremy W. Bachelor es doctorando en Educación por la Universidad Internacional Iberoamericana (México), máster en Lengua Espańola por la Universidad Estatal de Illinois y licenciado en Lengua Española por la Universidad de Eureka. Trabaja en el Centro Universitario Heartland (Heartland Community College) en Illinois. Contacto: Jeremy.Bachelor@heartland.edu
} 


\section{Intrinsic connection between pragmatics and grammar}

\section{Abstract}

Previous studies suggest that grammar is a prerequisite to Language 2 or second/ foreign language (L2) pragmatic acquisition, although high grammatical competence does not guarantee high pragmatic competence. This study more extensively examines the correlation between these two competencies. In the year 2013 at a North American university, 29 Spanish students at the intermediate-high level took a grammar test and a pragmatic test. The pragmatic test assessed requests, invitations, refusals, and apologies in Spanish. In comparing the two, the results suggest a positive relationship between pragmatic and grammatical competencies. Therefore, this research supports previous studies showing that the grammatical competence of students of foreign languages is necessary for the development of pragmatic competence; however, it is not sufficient for it in and of itself.

Keywords: pragmatics, grammar, Spanish as a foreign language, sociolinguistics, education

\section{Conexáo intrínseca entre pragmática e gramática}

\section{Resumo}

Muitos estudos sugerem que a gramática é um prérequisito para a aquisição da pragmática na Língua 2 ou segunda língua / língua estrangeira (L2), embora uma alta competência gramática não garante uma alta competência pragmática. Este estudo examina mais profundamente a correlação entre estas duas competências. Em 2013 em uma universidade americana, 29 estudantes espanhóis de nível medio-alto realizaram um teste gramatical e um teste de múltipla escolha sobre a pragmática. $\mathrm{O}$ teste de pragmática avaliou os usos comuns de solicitaçôes, convites, recusas e desculpas em espanhol. Comparando os dois, os resultados sugerem uma relação positiva entre a capacidade de competência gramatical e pragmática. Portanto, esta pesquisa apoia estudos anteriores que mostram que a competência gramatical de estudantes de línguas estrangeiras é necessaria para o desenvolvimento da competência pragmática; no obstante, não é suficiente por si só.

Palavras-chave: pragmática, gramática, espanhol como língua estrangeira, sociolinguística, educação 


\section{INTRODUCCIÓN}

Los profesores de español como lengua extranjera (ELE) han comenzado a entender la importancia de enseñar aspectos sociales del espańol, como la pragmática (Vellenga, 2011). La pragmática se interesa en analizar cómo los hablantes producen e interpretan enunciados en contexto (Martín Peris et al., 2008); no solamente abarca contextos, sino también inferencias y otros factores de la lengua con el fin de determinar el significado de la intención del hablante, lo cual varía de cultura a cultura. Además, debido al impulso reciente a nivel mundial para enseñar idiomas con la comunicación como objetivo principal (Poehner \& van Compernolle, 2011), la comprensión social y las señales culturales son más importantes que nunca (Roever, 2011). No obstante, no significa que la corrección gramatical no sea relevante, al contrario. Algunos investigadores han demostrado que la competencia pragmática de estudiantes de lenguas extranjeras depende de su competencia gramatical (Wedgwood, 2012). Ya que la competencia pragmática forma parte de la competencia global del estudiante de español, resulta necesario descubrir si esta depende del nivel gramatical del mismo, como señaló Wedgwood (2012).

Este artículo proveerá información básica sobre la pragmática en cuanto al estudio de lenguas extranjeras, incluirá un apartado sobre lagunas en las investigaciones actuales y la necesidad de un estudio de este tipo. Proporcionará una breve reseńa de los estudios anteriores en relación con la adquisición gramatical y pragmática en el aprendizaje de lenguas extranjeras con un enfoque en español, facilitará información sobre el diseño de la metodología y el estudio, y concluirá con una discusión de los resultados y las implicaciones pedagógicas más importantes.

\section{JUSTIFICACIÓN DEL ESTUDIO}

Este estudio intenta determinar la relación entre la gramática y la pragmática de los estudiantes de español como lengua extranjera. Ya se sabe que la gramática es necesaria para poder comunicarse, pero, además, la competencia pragmática es un componente necesario para una comunicación efectiva. Por ejemplo, V. G. y Rajan (2012) descubrieron que las habilidades de comunicación de los estudiantes no nativos se veían afectadas negativamente por la falta de conocimiento pragmático. Como el objetivo principal del aprendizaje de idiomas es la comunicación, la instrucción pragmática es necesaria para asegurar que los estudiantes son capaces de comunicarse de una manera efectiva. 
Estudios previos, como el de Håkansson y Norrby (2005), demostraron una relación entre las competencias gramaticales y pragmáticas en estudiantes de sueco; los alumnos con un alto nivel de gramática mostraron, por lo general, comandos pragmáticos parecidos a los de un hablante nativo y aquellos con niveles gramaticales más bajos demostraron habilidades pragmáticas pobres. Sin embargo, había casos aislados de estudiantes que no encajaban en esta generalización. El presente estudio busca validar los resultados de Håkansson y Norrby (2005), solo para estudiantes de ELE.

Es importante ver, además, si el desarrollo gramatical garantiza un nivel correspondiente de desarrollo pragmático. Algunos estudios (Hill, 1997; Roever, 2005; Yamashita, 1996) mostraron que los participantes con una competencia alta de gramática tuvieron un mejor desempeño en las pruebas de pragmática que los participantes de baja competencia lingüística en el contexto de inglés como segundo idioma. Por otro lado, otros estudios (Omar, 1991; Takahashi \& Beebe, 1987) mostraron disparidades entre el desarrollo gramatical de los alumnos y su desarrollo pragmático. El presente trabajo intenta llenar este vacío y determinar la relación entre la pragmática y la gramática en el contexto del español como lengua extranjera. Tal determinación sería de gran utilidad para profesores de español ya que tienen que determinar la forma de aplicar la gramática y pragmática en sus aulas, específicamente la cantidad de tiempo para cada una y si se les debe enseñar por separado o en conjunto.

Otra innovación con respecto a las investigaciones mencionadas anteriormente es el hecho de que se estudiaron solo características pragmáticas específicas. La investigación de la pragmática se ha centrado principalmente en la investigación de aspectos aislados de la competencia pragmática de estudiantes de idiomas. Muy pocos autores han intentado analizar más de un acto de habla en el mismo estudio. Para esta investigación, se analizó la producción de cuatro actos de habla diferentes (solicitud, invitación, rechazo y disculpa), para intentar llegar a una conclusión más global sobre el conocimiento pragmático de los estudiantes.

Por último, las razones personales que han motivado la elección de este tema han sido diversas. Al cursar asignaturas de postgrado, empecé a centrarme en la pragmática de las lenguas extranjeras. Siempre estuve interesado en la enseñanza de lenguas, la lingüística y los estudios culturales, y, en este sentido, la pragmática es una disciplina que combina a la perfección todas estas áreas. Como profesor universitario de español, me he dado cuenta de las tendencias en el discurso de mis estudiantes en relación con la gramática y la pragmática. Parece que los estudiantes que triunfan en las lecciones gramaticales tienden a adquirir la pragmática con más facilidad que los estudiantes que carecen 
de competencia gramatical. Estoy muy interesado en descubrir la conexión entre las dos competencias, con el fin de agregar valor adicional a mis lecciones gramaticales incluyendo contenidos pragmáticos.

\section{EsTADO DE LA CUESTIÓN}

Según Romero Betancourt (2012), la pragmática es un área de investigación que se centra en los actos lingüísticos dentro de contextos. En el ámbito de la enseñanza de lenguas extranjeras, la instrucción pragmática intenta facilitar la capacidad de los alumnos para relacionar el uso socialmente adecuado del idioma dentro de los contextos en los que se encuentran (V.G. y Rajan, 2012). V.G. y Rajan explican que la pragmática abarca los actos del habla, la estructura conversacional, la implicatura conversacional, la gestión conversacional, la organización del discurso y los aspectos sociolingüísticos del uso del idioma, así como la elección de las formas de trato.

A lo largo de la adquisición del dominio de una lengua extranjera, existe un sistema conocido como interlengua (Schulz, 2011), que es un tipo de sistema lingüístico utilizado por estudiantes de idiomas extranjeros para almacenar nuevos conocimientos adquiridos. Durante una cierta etapa de la interlengua, el alumno llega a conocer los matices en el uso del lenguaje; los matices específicos a menudo tienen que ver con la competencia pragmática.

Es más, la competencia pragmática es un componente necesario de la comunicación efectiva. Durante muchos años, los profesores de lenguas extranjeras centraron su atención en la competencia gramatical y los métodos de enseñanza que aplicaban se basaban en ahondar en la gramática y la memorización (Bachelor, 2013). Sin embargo, un cambio en el horizonte se ha producido en los últimos años y los investigadores han descubierto que el principal objetivo de la enseñanza de lenguas extranjeras tiene que ver con una comunicación efectiva (Poehner \& van Compernolle, 2011).

V.G. y Rajan (2012) descubrieron que las habilidades de comunicación de los estudiantes no nativos se vieron afectadas negativamente por la falta de conocimiento pragmático. Bajo la premisa de que la comunicación es el objetivo principal del aprendizaje de idiomas, el estudio de V.G. y Rajan sugiere que la instrucción pragmática es necesaria para asegurar que los estudiantes sean capaces de comunicarse de una manera efectiva y que la instrucción únicamente centrada en la gramática no es suficiente.

$\mathrm{Al}$ investigar la pragmática, Bataller (2008) encontró que un bajo nivel de competencia gramatical restringió la capacidad pragmática de los estudiantes. 
Sugiere que los estudiantes con una competencia gramatical baja no son capaces de producir enunciados pragmáticos adecuados debido a la falta de un fundamento lingüístico. En un estudio similar, Schauer (2009) descubrió una relación clara entre las competencias gramaticales y pragmáticas; los alumnos con un alto nivel de gramática mostraron, por lo general, comandos pragmáticos similares a los de un nativo hablante y aquellos con niveles gramaticales más bajos demostraron habilidades pragmáticas pobres. Ella sí encontró casos en los que los estudiantes con alta competencia gramatical mostraron baja competencia pragmática, lo que sugiere que la pragmática depende de la gramática, pero aún debe ser adquirida como una entidad separada dentro de la interlengua.

Todos estos estudios anteriores dan una idea de la conexión que la gramática y pragmática tienen en el aprendizaje del idioma español; sin embargo, esta idea no es muy precisa. Si bien la literatura sobre el aprendizaje de lenguas extranjeras en general sugiere una conexión, los profesores de español deben saber si esto es aplicable también a su campo de estudio y para sus alumnos.

\subsection{Las investigaciones sobre la enseñanza de la pragmática}

Un breve resumen de las últimas metodologías de enseñanza de lenguas extranjeras es necesario para comprender por qué la instrucción pragmática se ha convertido en un componente importante de la enseńanza de lenguas. Hasta principios de la década de 1970, los profesores de lenguas extranjeras se ocupaban principalmente de la forma lingüística, algo reflejado en los enfoques generativos como el método gramática-traducción (Chang, 2011). A través de ejercicios de traducción y presentación de la gramática, se alentaba a los estudiantes a desarrollar la competencia gramatical mediante la memorización de las conjugaciones verbales y otros paradigmas lingüísticos (Larsen-Freeman, 2000). Otros métodos que se centraron en la perfección de la gramática se han empleado a lo largo de los ańos, tales como el método directo y el método audiolingual. Sin embargo, los educadores pronto comenzaron a reconocer la falta de atención hacia otros componentes lingüísticos esenciales, como los de hablar y escuchar (Brown, 2006). El método de lenguaje comunicativo surgió como marco principal y, desde entonces, ha sido adoptado por los investigadores del lenguaje (Larsen-Freeman, 2000). En lugar de centrarse en el desarrollo de las expresiones gramaticales perfectas a través de la memorización, se enseña a los estudiantes que se aprende una lengua extranjera como medio para comunicarse (Morrow, 1981). La comunicación efectiva se convirtió en el objetivo principal del aprendizaje de lenguas extranjeras. Además, se ha descubierto 
que las normas sociolingüísticas, como la pragmática, son componentes esenciales de una comunicación efectiva y que la falta de competencia pragmática influye negativamente en la capacidad global del estudiante para comunicarse (V.G. y Rajan, 2012).

Bardovi-Härlig (2001), Rose y Kasper (2001), Schmidt (1995), entre otros, han examinado las aplicaciones pedagógicas de la pragmática. Una de las principales áreas de estudio ha sido la hipótesis de la captación (o noticing) y la función de aducto (o input). Ha habido cierto debate sobre si esos conceptos implícitos funcionan mejor que la instrucción explícita (Alcón Soler, 2005; Norris \& Ortega, 2000). Los investigadores están divididos entre los que creen que sí hay una «interfaz» entre los procesos implícitos y explícitos (DeKeyser, 2005), y los que opinan que no la hay en absoluto (Krashen, 1985; Ellis, 1994). Mir (2001) explica que, aunque es probable que la instrucción implícita de la pragmática pueda funcionar, el estudiante tiene que estar en contacto con una comunidad de hispanohablantes o pasar horas en el aula de clase. La autora propone la implementación de una instrucción pragmática más activa y explícita para que los estudiantes puedan interiorizar reglas e incorporarlas activamente en su uso del idioma. Langer (2011) coincide en su estudio sobre la instrucción pragmática explícita, pues la competencia pragmática de sus estudiantes mejoró significativamente en todos los ámbitos después de sus intervenciones explícitas. Una enseñanza explícita y activa, explicada por Mir (2001), es la que se recomienda en el último apartado de este trabajo para los profesores de ELE.

A pesar de los estudios sobre la importancia de la pragmática, muchos profesores de ELE no ven valor en su enseñanza y tampoco están formados en su instrucción. Los resultados de esta investigación podrían inspirar a dichos profesores, ya que verían que la inclusión de la pragmática en sus desarrollos curriculares puede ir de la mano con la instrucción gramatical, estableciendo una relación sinérgica.

\subsection{La interacción entre el desarrollo pragmático y gramatical}

Es importante explorar las teorías de otros expertos que han investigado este tema y, después, aplicar esas ideas al presente estudio para profesores de ELE. Como se ha ido viendo, algunos sostienen que la competencia gramatical es un requisito previo a la competencia pragmática (Håkansson \& Norrby, 2005; Ran, 2007; Luo \& Gao, 2011); sin embargo, hay estudios que indican lo contrario. Por ejemplo, Kasper y Rose (2001) sugieren que los estudiantes de idiomas adquieren una considerable cantidad de conocimiento pragmático de la lengua extranjera a través de su lengua materna, ya que, según ellos, 
muchos conocimientos pragmáticos son universales y pueden ser transferidos desde la primera lengua a la lengua extranjera, como es el caso de algunos actos comunicativos específicos, la implicatura conversacional, la variabilidad sociopragmática y la elección lingüística. Llegan a estas conclusiones haciendo referencia frecuente al estudio de Schmidt (1995), quien analizó meticulosamente el caso de un adulto que demostró poca precisión gramatical, pero aun así fue capaz de interpretar los mensajes destinados y de producir enunciados pragmáticamente adecuados.

Sin embargo, Ishihara y Cohen (2010) explican que la capacidad gramatical de los estudiantes tiene un impacto sobre su competencia pragmática. Los estudiantes comprenden las intenciones pragmáticas mejor cuando incluyen estructuras gramaticales que les son familiares. Del mismo modo, los estudiantes solo son capaces de producir estructuras gramaticales que han aprendido. Por ejemplo, un estudiante que no estuviese familiarizado con oraciones compuestas no las comprendería al escucharlas ni las podría producir, lo cual limita su alcance pragmático (Bardovi-Härlig, 2003). Parece que, aunque ha habido casos aislados de estudiantes que han logrado una competencia pragmática alta con baja competencia gramatical, la tendencia es que la gramática para el aprendizaje del inglés es un requisito previo para la adquisición pragmática. Los estudiantes que adquirieren la pragmática, ya sea implícita o explícitamente, simplemente no serían capaces de expresarse sin una base gramatical, lo que resultaría en un fallo pragmático. Esta investigación se basa precisamente en esa idea, aunque busca más datos en cuanto a la adquisición del español.

\subsection{Los componentes dentro de la competencia lingüística}

Todo lo anterior quizá ya tenía fundamento en las teorías de Canale y Swain (1980) y de Chomsky (1965), ya que los estudios sobre la relación entre la competencia gramatical y la competencia pragmática comenzaron primero con el inicio del enfoque de competencia comunicativa en la enseñanza de lenguas extranjeras (Canale \& Swain, 1980), aunque no hablaron explícitamente de la pragmática. Se identificaron cuatro ámbitos de uso de la lengua que los estudiantes de lenguas extranjeras necesitan para ser globalmente competentes: la competencia gramatical, el ámbito sociocultural (la pragmática), el discursivo, y el estratégico. No obstante, el enfoque no contiene ninguna referencia al orden de adquisición.

Varios modelos influyentes actuales sobre la capacidad comunicativa de la lengua representan el conocimiento de idiomas como un conjunto de componentes relacionados pero separados, incluyendo dimensiones gramaticales y 
pragmáticas, que interactúan con el conocimiento previo, estrategias metacognitivas y características contextuales en el uso del lenguaje (Chapelle, 1998).

Según Chomsky (1965), la competencia lingüística exige que el locutor utilice el lenguaje no solo correctamente, sino también de una manera apropiada. Hymes (1972) coincide con esta idea, y afirma que un niño adquiere el conocimiento de frases no solo como algo gramatical, «but also as appropriate» [sino también como algo apropiado] (p. 277). Un niño llega a ser capaz de lograr un repertorio de actos de habla y de tomar parte en eventos de habla.

Estos enfoques no disminuyen la importancia de aprender las reglas gramaticales de una lengua. De hecho, es uno de los cuatro componentes de la competencia comunicativa. Aunque, por supuesto, la adquisición formal de la lengua también es posible por proceso natural, según Chomsky (1965).

Todo esto indica que la competencia comunicativa es una combinación de todas las competencias lingüísticas, lo que indica que hay que enseñar tanto la gramática como la pragmática en las clases de ELE con el fin de producir un alto nivel de competencia global.

\section{Metodología}

Debido a las disparidades entre la relación de esas dos competencias en cuanto al español, es importante seguir investigando este tema. En el cuatrimestre de otońo de 2013 en una universidad en las afueras de Chicago, 29 estudiantes que cursaron asignaturas de español como lengua extranjera a nivel intermedio-alto o avanzado hicieron la prueba de gramática española de la Universidad de Oxford y un cuestionario de elección múltiple (MCDCT) sobre el nivel pragmático.

\subsection{El diseño}

Para empezar el cuatrimestre, se eligieron tres grupos de estudiantes. Los tres grupos cursaban tres asignaturas diferentes, siendo todas ellas de nivel intermedio-alto o avanzado, según la categorización de la universidad en cuestión. Todos dieron consentimiento al participar y, después, los tres grupos hicieron una prueba para determinar su nivel de competencia gramatical y otra prueba para su nivel pragmático. El diseño de esta investigación es cuantitativo. Según Merrill (2011), el objetivo para la investigación cuantitativa es determinar la relación entre dos variables en una población. Además, como no hay tratamiento después de las pruebas, el diseño no es experimental. 


\subsection{Preguntas de investigación}

Las preguntas de investigación del estudio son:

(1) ¿Hay alguna relación entre la competencia gramatical y la pragmática de los estudiantes de español?

(2) ¿Es la competencia gramatical de los estudiantes de español necesaria para la competencia pragmática?

(3) ¿Es la competencia gramatical suficiente para adquirir competencia pragmática?

\subsection{Población}

La población del estudio son todos los estudiantes que cursaron asignaturas de español como lengua extranjera a nivel intermedio-alto o avanzado, en la universidad mencionada, durante el cuatrimestre de otońo de 2013. Según la secretaria del Departamento de Lenguas Modernas de la universidad en cuestión, había 80 estudiantes cursando asignaturas de ELE a nivel intermedio-alto o avanzado en el otońo de 2013. La petición para participar en el estudio fue enviada a los 80 estudiantes en cuestión y 29 de ellos participaron. Según Gutzman (2013), los estudios cuantitativos dentro del entorno educativo requieren una fórmula que use datos donde los valores puedan cambiar de forma continua. El autor especifica que una población de 80 estudiantes y una muestra de 29 estudiantes tiene una tasa de confianza del $90 \%$ y un margen de error del $12.2 \%$.

Los participantes en la muestra son estudiantes universitarios y varían en gran medida en su campo de estudio. Tienen entre 18 y 22 ańos de edad, son principalmente caucásicos y provienen de familias de clase media y alta. No hubo parcialidad en la selección de los grupos. El investigador no tuvo acceso a información sobre los grupos a la hora de seleccionarlos. El proceso de selección se basó en las características anteriores descritas.

La escena en la que se llevó a cabo el estudio es una universidad privada en las afueras de una gran área metropolitana en el medio oeste de los Estados Unidos. El barrio residencial es pequeño, con alrededor de 15 mil habitantes, aunque el área metropolitana tiene más de 9,5 millones de residentes. La universidad en cuestión tiene más de 4000 estudiantes y el índice hombre-mujer es comparable con el promedio nacional de aproximadamente 40:60, con un cuerpo estudiantil predominantemente femenino. El 80\% de los estudiantes tiene entre 18 y 22 años de edad. El 83\% de la población universitaria es caucásico, el $11 \%$ es afroamericano y el $4 \%$ es hispano. 
La puntuación promedio de la prueba de acceso a la universidad (ACT) del estudiante admitido es de 24 , un punto por encima de la media nacional de los estudiantes admitidos a otras universidades. La universidad está acreditada y reconocida oficialmente y ofrece títulos de grado en 67 campos de estudio, títulos de máster y un título de doctorado. Los cinco mayores campos de estudio son enfermería, administración de empresas, educación, arte y trabajo social (U.S. News y World Report, 2013).

\subsection{Instrumentos de investigación}

Los métodos de investigación incluyen:

(1) Una prueba de gramática de español

(2) Una prueba de pragmática de español

La prueba de gramática de español es la prueba de nivel de la Universidad de Oxford. Se trata de una evaluación virtual de opción múltiple que calcula el conocimiento gramatical y el vocabulario de los estudiantes. Está diseñada para los estudiantes mayores de 15 años y los temas y las situaciones son apropiados para gente de esa edad en adelante. Las pruebas de nivel de gramática que ofrece la Universidad de Oxford han sido previamente realizadas por más de 19000 estudiantes en 60 países. La Universidad de Oxford utilizó los resultados de las pruebas preliminares para eliminar o hacer cambios necesarios. La prueba es fácil de administrar y es una manera muy práctica de recolectar datos válidos en el conocimiento gramatical de los alumnos de una lengua extranjera (Oxford University Press, 2013). Los resultados están disponibles de inmediato y las puntuaciones se dividen en cinco niveles: principiante, intermedio bajo, intermedio, intermedio alto y avanzado (Oxford University Language Centre, 2010).

La prueba de pragmática de español es una versión modificada del cuestionario de producción (DCT) de la tesis doctoral de Langer (2011) y ha sido convertida en un cuestionario tipo test (MCDCT). El cuestionario se compone de veinte situaciones que requieren que el participante elija una solicitud, una invitación, un rechazo y una disculpa. Estos cuatro actos de habla fueron seleccionados debido a que tienden a ser las acciones más estudiadas y son las menos bruscas (face-threatening) (Langer, 2011). Aunque el MCDCT requiere que los estudiantes seleccionen una respuesta en español, todas las instrucciones y escenarios están escritos en inglés para asegurar la comprensión total de los estudiantes, lo cual es especialmente importante, ya que no todos los sujetos tienen un nivel superior de español. De esta manera se reduce la posibilidad de malentendido a lo largo del proceso de recolección de datos. 
El MCDCT en general es un mecanismo flexible para la recogida de datos y la evaluación de la competencia pragmática en muchos aspectos (Linde, 2009). Liu (2007) desarrolló un MCDCT para valorar el conocimiento pragmático de estudiantes chinos de inglés en relación con el acto de disculparse y su estudio proporciona evidencia de la fiabilidad y validez de la prueba. Entre los estudios comparativos sobre los varios instrumentos para evaluar la pragmática, Jianda (2006) comparó la viabilidad del cuestionario de producción (DCT), el cuestionario de elección múltiple (MCDCT) y los juegos de rol (DRPT). Se estimó la consistencia interna de cada prueba y las estimaciones de la fiabilidad del DCT y del DRPT fueron de 0,90, mientras que para el MCDCT fue de 0,86 , lo que demostró que las tres pruebas tenían una alta fiabilidad.

Debido a que el MCDCT ha sido validado por varias investigaciones, el presente estudio lo ha seleccionado como el instrumento más adecuado, ya que es cómodo de administrar, se tarda muy poco en llevar a cabo, es fácil analizar el rendimiento de los estudiantes y no hay ninguna preocupación acerca de la confiabilidad entre evaluadores (Ahn, 2005). Se reconoce que este instrumento evalúa la habilidad del estudiante para reconocer la pragmática en vez de producirla, pero los participantes en este estudio son estudiantes de español como lengua extranjera y es importante entender su nivel de pragmática sin presentar barreras gramaticales a las que se enfrentarían si tuvieran que escribir sus respuestas a mano.

Como el instrumento de Langer (2011) ha sido modificado, era importante comprobar su validez. Por este motivo, el instrumento fue enviado a diez nativohablantes de español para ver su opinión y para verificar que las opciones que se plantean en el instrumento corresponden con lo que un hablante nativo probablemente diría en un contexto real. Además, esta prueba sirve para justificar por qué esas son las opciones correctas que los hablantes no nativos deberían escoger. Se les dio a los participantes hispanohablantes la opción de poner otra respuesta que les pareciera más adecuada o natural, y la opción de aportar sus comentarios en el caso de que las opciones propuestas no les resultaran correctas.

A continuación se presenta un ejemplo de una de las preguntas del MCDCT:

You ask your friend if you can use his/her Spanish textbook [Preguntas a un amigo tuyo si puedes usar su libro de texto de español].

You say [Tú dices]:

A. ¿Me puedes dar el libro?

B. Quiero tener tu libro, por favor.

C. Por favor, dame tu libro. 


\section{ANÁlisis de los datos}

Los resultados sobre las competencias lingüísticas y pragmáticas de los participantes fueron respectivamente recogidos, y se presentan en los siguientes gráficos. La prueba de pragmática contiene 20 preguntas; cada una de las cuales vale 5 puntos dando un total de 100 puntos. Para cada pregunta contestada incorrectamente, el estudiante pierde 5 puntos. Las puntuaciones totales de cada estudiante se reflejan en porcentajes. Por su parte, la de gramática contiene 50 preguntas y cada una de ellas vale 1 , con un posible máximo de 50 puntos. Los puntos obtenidos por el estudiante se dividieron entre 50, con el objetivo de encontrar el porcentaje. Tanto ambos porcentajes para la pragmática como para la gramática se representan en la Tabla 1.

Tabla 1. Resultados de las pruebas pragmáticas y gramaticales

\begin{tabular}{lccl}
\hline Estudiante & Pragmática & Gramática & Nivel pragmático / gramatical \\
\hline Estudiante 1 & $65 \%$ & $44 \%$ & Intermedio / Intermedio bajo \\
Estudiante 2 & $55 \%$ & $58 \%$ & Intermedio / Intermedio \\
Estudiante 3 & $60 \%$ & $52 \%$ & Intermedio / Intermedio \\
Estudiante 4 & $35 \%$ & $40 \%$ & Intermedio bajo / Intermedio bajo \\
Estudiante 5 & $55 \%$ & $78 \%$ & Intermedio / Intermedio alto \\
Estudiante 6 & $35 \%$ & $32 \%$ & Intermedio bajo / Intermedio bajo \\
Estudiante 7 & $55 \%$ & $76 \%$ & Intermedio / Intermedio alto \\
Estudiante 8 & $80 \%$ & $82 \%$ & Intermedio alto / Intermedio alto \\
Estudiante 9 & $45 \%$ & $54 \%$ & Intermedio bajo / Intermedio \\
Estudiante 10 & $60 \%$ & $68 \%$ & Intermedio / Intermedio \\
Estudiante 11 & $60 \%$ & $74 \%$ & Intermedio / Intermedio alto \\
Estudiante 12 & $55 \%$ & $82 \%$ & Intermedio / Intermedio alto \\
Estudiante 13 & $35 \%$ & $62 \%$ & Intermedio bajo / Intermedio \\
Estudiante 14 & $70 \%$ & $44 \%$ & Intermedio / Intermedio bajo \\
Estudiante 15 & $50 \%$ & $60 \%$ & Intermedio / Intermedio \\
Estudiante 16 & $60 \%$ & $70 \%$ & Intermedio / Intermedio \\
Estudiante 17 & $60 \%$ & $62 \%$ & Intermedio / Intermedio \\
Estudiante 18 & $75 \%$ & $56 \%$ & Intermedio alto / Intermedio \\
Estudiante 19 & $25 \%$ & $68 \%$ & Principiante absoluto / Intermedio \\
Estudiante 20 & $45 \%$ & $44 \%$ & Intermedio bajo / Intermedio bajo \\
Estudiante 21 & $35 \%$ & $64 \%$ & Intermedio bajo / Intermedio \\
& & &
\end{tabular}




\begin{tabular}{lccl}
\hline Estudiante & Pragmática & Gramática & Nivel pragmático / gramatical \\
\hline Estudiante 22 & $45 \%$ & $90 \%$ & Intermedio bajo / Intermedio alto \\
Estudiante 23 & $45 \%$ & $74 \%$ & Intermedio bajo / Intermedio alto \\
Estudiante 24 & $40 \%$ & $78 \%$ & Intermedio bajo / Intermedio alto \\
Estudiante 25 & $55 \%$ & $60 \%$ & Intermedio / Intermedio \\
Estudiante 26 & $60 \%$ & $66 \%$ & Intermedio / Intermedio \\
Estudiante 27 & $60 \%$ & $64 \%$ & Intermedio / Intermedio \\
Estudiante 28 & $50 \%$ & $48 \%$ & Intermedio/ Intermedio bajo \\
Estudiante 29 & $40 \%$ & $50 \%$ & Intermedio bajo/ Intermedio \\
\hline Total & $52 \%$ & $62 \%$ & Intermedio / Intermedio \\
\hline
\end{tabular}

Se pueden observar en la Tabla 1 los resultados y sus niveles correspondientes según las categorías establecidas por la prueba de nivel de la Universidad de Oxford.

Antes de su conversión en porcentaje, la puntuación para la prueba de gramática original se colocó en una de las siguientes cinco categorías, según la escala de la Universidad de Oxford: Principiante absoluto, 0-12; Intermedio bajo, 13-24; Intermedio, 25-36; Intermedio alto, 37-46; y Avanzado, 47-50. Los porcentajes de cada estudiante de la prueba de pragmática fueron entonces multiplicados por 0,5 para dar a cada estudiante una puntuación de 0 a 50 , con el fin de ponerlas en las mismas categorías ofrecidas por la Universidad de Oxford. Los promedios se proporcionan al final de la Tabla 1.

Trece de los estudiantes tenían el mismo nivel pragmático que gramatical. 25 de los estudiantes tenían un nivel gramatical más alto que su nivel pragmático. Y 4 de los estudiantes tenían un nivel pragmático más alto que su nivel gramatical.

Figura 1. Niveles de gramática y pragmática

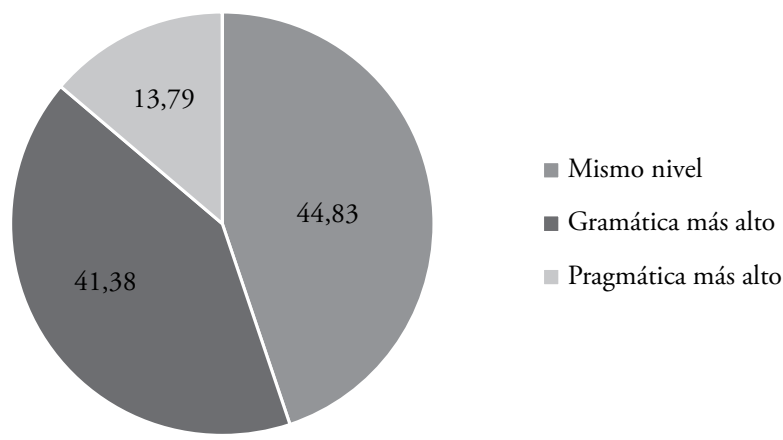

Fuente: Elaboración propia de los resultados. 


\section{Discusión}

La Tabla 1 y la Figura 1 sugieren que existe una relación positiva entre la habilidad pragmática y la competencia gramatical entre estudiantes de ELE. Los participantes con alta competencia pragmática, en general, tienen la capacidad gramatical alta o incluso superior. Por tanto, esta investigación apoya estudios previos sobre la adquisición del inglés que demuestran que la competencia gramatical de estudiantes de lenguas extranjeras es necesaria para el desarrollo de la competencia pragmática.

Aun así, el 13,79\% de los participantes tenían un nivel pragmático mayor que su nivel gramatical. Estos datos contradicen muchos estudios que aseguran que la gramática es necesaria para la pragmática.

$\mathrm{Ni}$ un solo participante logró una calificación avanzada en las pruebas de gramática o pragmática. Un gran porcentaje (el 44,83\%) logró el mismo nivel, tanto en la gramática como en la pragmática. El 41,37\% de los estudiantes logró un nivel superior de gramática que de pragmática.

Este estudio indica lo que muchos investigadores han contrastado: la competencia gramatical es un requisito previo a la competencia pragmática (Bardovi-Härlig, 2003; Håkansson \& Norrby, 2005; Ran, 2007; Ishihara \& Cohen, 2010; Luo \& Gao, 2011). La mayoría de los estudiantes con un nivel intermedio de gramática también tenía un nivel intermedio de pragmática.

Como muestra la Figura 1, existe todavía un importante porcentaje de participantes que obtuvo calificaciones más altas en la prueba de pragmática que en la prueba de gramática. Las variables globales para los resultados están relacionadas, aunque no muy relacionadas, lo que sugiere que la competencia gramatical no es suficiente para la capacidad pragmática en estudiantes de ELE. Por tanto, la conclusión preliminar que puede extraerse es que la competencia gramatical no es el único factor para determinar la capacidad pragmática. Kasper y Rose (2001), por ejemplo, sugieren que los estudiantes de idiomas adquieren una considerable cantidad de conocimiento pragmático de su lengua materna porque, según ellos, muchos conocimientos pragmáticos son universales y pueden ser transferidos desde la primera lengua a la segunda lengua.

\section{CONCLUSIONES, LIMITACIONES E IMPLICACIONES PEDAGÓGICAS}

Una de las limitaciones de este estudio consiste en la baja tasa de participación en las pruebas. Solo el 36\% de los estudiantes de nivel intermedio-alto y avanzado participaron en el estudio, tal vez debido a las fechas del estudio, 
ya que coincidió con las fechas de los exámenes finales. Aun así, el tamaño de la muestra es representativo para el propósito de este trabajo, aunque se indica la necesidad de investigaciones más amplias para ahondar en los objetivos principales de esta investigación.

Otra limitación encontrada es el diseño de la prueba de pragmática. Mientras que el formato de opción múltiple para las pruebas de pragmática ha sido utilizado con éxito por muchos investigadores en el pasado (Jianda, 2006; Linde, 2009; Liu, 2007; Roever, 2005), Ahn (2005) explica que algunos estudiantes pueden seleccionar las respuestas descuidadamente y que, a veces, es difícil para los hablantes nativos decidir la respuesta más adecuada y no hay producción de lenguaje real, sino solo reconocimiento. Como los estudiantes estaban ocupados con los exámenes finales, es posible que el 13,79\% de los estudiantes que lograron una puntuación más alta en pragmática que en gramática simplemente eligieran cualquier respuesta o tuvieran suerte al adivinar la respuesta.

Este estudio sugiere que la competencia gramatical es necesaria para la competencia pragmática en estudiantes de ELE, pero que no es suficiente para adquirir un dominio de la lengua. En otras palabras, la competencia gramatical es un requisito previo necesario para la competencia pragmática, pero no garantiza la competencia pragmática. Futuras investigaciones pueden centrarse en las diferencias entre el conocimiento pragmático y gramatical de los estudiantes de español con diferentes competencias lingüísticas. Se necesitan más estudios que comparen los niveles pragmáticos y gramaticales antes y después de intervenciones por parte de profesores o investigadores. El entorno perfecto para un estudio de este tipo sería el aula de clase de un idioma extranjero, donde los estudiantes todavía están aprendiendo la gramática en un nivel inicial o intermedio bajo. Futuras investigaciones permitirán responder estas preguntas.

Las implicaciones pedagógicas de los resultados de este estudio son importantes. Antes de esta investigación existía un vacío de conocimiento sobre la posible conexión entre la pragmática y la gramática en estudiantes de ELE y, por eso, una gran cantidad de profesores de lenguas extranjeras no veían valor en la enseñanza de la pragmática o no estaban formados en su instrucción. Las implicaciones pedagógicas de la presente investigación deberían tener un impacto sobre esos profesores, ya que los resultados demuestran que la instrucción pragmática es necesaria y que los estudiantes, aunque requieren de una base gramatical, no adquieren la pragmática sin lecciones sobre ella o sin una experiencia en el extranjero.

Asimismo, se recomienda una instrucción explícita y activa de la pragmática en las clases de ELE. Como indica Mir (2001), la mejor manera de 
aprender la pragmática es por un proceso natural; el problema es que los estudiantes tendrían que pasar una considerable suma de tiempo en un país hispanohablante para adquirir la pragmática de manera implícita. Por eso, existen instrucciones explícitas que luego son seguidas por actividades activas, que se incorporan a actividades ya existentes que usan tanto el vocabulario como la gramática estudiados. Estas actividades deben alinearse a los principios del método comunicativo en el sentido de que todo lo que hacen los estudiantes tiene que tener un enfoque en el significado y en la vida práctica del estudiante.

Por último, los profesores de ELE continuarán diseñando planes de estudio para cumplir con sus programas y los resultados de aprendizaje, pero la conclusión de este estudio verifica que la pragmática es necesaria. No solo eso, sino que hay efectos adicionales en la creación de planes de estudio que se presten a la aplicación en el mundo real, que es precisamente lo que hacen las lecciones pragmáticas. Es una responsabilidad primordial como educadores de ELE encontrar y desarrollar lecciones efectivas para los estudiantes. Por ello, la instrucción pragmática debe ser altamente considerada.

\section{REFERENCIAS BIBLIOGRÁFICAS}

Ahn, R. C. (2005). Five measures of interlanguage pragmatics in KFL (Korean as a foreign language) learners (tesis sin publicar). University of Hawaii, Hawái, Estados Unidos.

Alcón Soler, E. (2005). Does instruction work for learning pragmatics in the EFL context? System: An International Journal of Educational Technology and Applied Linguistics, 33, 417-435.

Bachelor, J. W. (2013). Sinergia entre las TIC y los sistemas de gestión de aprendizaje en el aula ELE presencial. Quaderns Digitals, 75.

Bardovi-Härlig, K. (2001). Evaluating the empirical evidence: Grounds for instruction in pragmatics? En K. Rose y G. Kasper (Eds.), Pragmatics in language teaching (pp. 13-32). Cambridge, Inglaterra: Cambridge University Press.

Bardovi-Härlig, K. (2003). Understanding the role of grammar in the acquisition of L2 pragmatics. Pragmatic Competence and Foreign Language Teaching, 25-44.

Bataller, R. (2008). Pragmatic development in a study abroad setting: Requesting a service in Spanish (tesis sin publicar). University of Iowa, Estados Unidos.

Brown, H. (2006). Principles of foreign teaching and learning. Hillsdale, NJ: L. Erlbaum Associates. 
Canale, M. \& Swain, M. (1980). Theoretical bases of communicative approaches to second language teaching and testing. Applied Linguistics, 1, 1-47.

Chang, S. (2011). A contrastive study of grammar translation method and communicative approach in teaching English grammar. English Language Teaching, 4(2), 13-24.

Chapelle, C. (1998). Construct definition and validity inquiry in SLA research. En L. Bachman y A. Cohen (Eds.), Interfaces between second language acquisition and language testing research (pp. 141-155). Cambridge, Reino Unido: Cambridge University Press.

Chomsky, Noam (1965). Aspects of the theory of syntax. Cambridge, MA: MIT. Press.

DeKeyser, R. M. (2005). What makes learning second-language grammar difficult? A review of issues. Language Learning, 55(1), 1-25.

Ellis, R. (1994). A theory of instructed second language acquisition. En N.C. Ellis (Ed.), Implicit and explicit learning of languages (pp. 79-114), San Diego, California: Academic.

Gutzman, D. (2013). Survey random sample calculator. Recuperado de http:// www.custominsight.com/articles/random-sample-calculator.asp

Håkansson, G., y Norrby, C. (2005). Grammar and pragmatics: Swedish as a foreign language. EUROSLA Yearbook, 5, 37-6.

Hill, T. (1997). The development of pragmatic competence in an EFL context (tesis sin publicar). Temple University, Tokio.

Hymes, D. H. (1972). On communicative competence. En J. B. Pride y J. Holmes (Eds.), Sociolinguistics: selected readings (pp. 269-293). Harmondsworth, Inglaterra: Penguin.

Ishihara, N., y Cohen, A. D. (2010). Teaching and learning pragmatics: Where language and culture meet. Harlow, Inglaterra: Pearson Longman.

Jianda, L. (2006). Assessing EFL learners' interlanguage pragmatic knowledge: Implications for testers and teachers. Reflections on English Language Teaching, 5(1), 1-22.

Kasper, G. \& Rose, K. R. (2001). Pragmatics in language teaching. En K. Rose y G. Kasper (Eds.), Pragmatics in language teaching (pp. 1-12). Cambridge, MA: Cambridge University Press.

Krashen, S.D. (1985). The input hypothesis: Issues and implications. New York: Longman.

Langer, B. D. (2011). The effects of pragmatic instruction in the Spanish language classroom (tesis sin publicar). University of California, California, Estados Unidos. 
Larsen-Freeman, D. (2000). Techniques and principles in language teaching. Oxford, Inglaterra: Oxford University Press.

Linde, A. (2009). How polite can you get?: A comparative analysis of interlanguage pragmatic knowledge in Spanish and Moroccan EFL university students. Porta Linguarum, 12, 133-147.

Liu, J. D. (2007). Developing a pragmatics test for Chinese EFL learners. Language Testing, 24(3), 391-415.

Luo, X. \& Gao, J. (2011). On pragmatic failures in second language learning. Theory and Practice in Language Studies, 1(3), 283-286.

Martín Peris, E., Atienza Cerezo, E., Cortés Moreno, M., González Arguello, V., López Ferrero, C. \& Torner Castells, S. (2008). Diccionario de términos clave de ELE. Madrid, Espańa: SGEL.

Merrill, K. C. (2011). Developing an effective quantitative research proposal. The Journal of Infusion Nursing, 34(3), 181-186.

Mir, M. (2001). Un modelo didáctico para la enseńanza de la pragmática. Hispania, 84(3), 542-549.

Morrow, K. (1981). Principles of communicative methodology. En K. Johnson y K. Morrow (Eds.), Communication in the Classroom (pp. 59-66) London, Inglaterra: Longman.

Norris, J. M. \& Ortega, L. (2000). Effectiveness of L2 instruction: A research synthesis and quantitative meta-analysis. Language Learning, 50, 417-528.

Omar, A. S. (1991). How learners greet in Kiswahili. En L. Bouton y Y. Kachru (Eds.), Pragmatics and language learning 2 (pp. 59-73). Urbana-Champaign, IL: University of Illinois.

Oxford University Language Centre (2010). Spanish placement test. (J. W. Bachelor, Trad.). Obtenido de http://www.lang.ox.ac.uk/courses/tst_placement_ spanish.html

Oxford University Press (2013). The Oxford online placement test. Obtenido de https://elt.oup.com/catalogue/items/global/exams_testing/97801945715 $48 ? \mathrm{cc}=$ usyselLanguage $=\mathrm{en}$

Poehner, M. E. \& van Compernolle, R. A. (2011). Frames of interaction in dynamic assessment: Developmental diagnoses of second language learning. Assessment in Education: Principles, Policy y Practice, 18(2), 183-198.

Ran, L. (2007). The relationship between linguistic proficiency and pragmatic ability. US-China Foreign Language, 5, 13-17.

Roever, C. (2005). Testing ESL pragmatics. Frankfurt, Alemania: Gunter Narr.

Roever, C. (2011). Testing of second language pragmatics: Past and future. Language Testing, 28(4), 463-481. 
Romero Betancourt, M. V. (2012). Adquisición de pragmática en segunda lengua. Un modelo didáctico para la enseñanza de la pragmática (tesis sin publicar). Indiana University, Indiana, Estados Unidos.

Rose, K. R. \& Kasper, G. (2001). Pragmatics in language teaching. Cambridge, Inglaterra: Cambridge University Press.

Schauer, G. A. (2009). Interlanguage pragmatic development: The study abroad context. Londres, Inglaterra: Continuum.

Schmidt, R. (1995). Consciousness and foreign language learning: A tutorial on the role of attention and awareness in learning. En R. Schmidt (Ed.), Attention and awareness in foreign language learning (pp. 1-63). Honolulu, HI: University of Hawai' i.

Schulz, B. (2011). Syntactic creativity in second language English: wh-scope marking in Japanese-English interlanguage. Second Language Research, 27, 313-341.

Takahashi, T. \& Beebe, L. (1987). The development of pragmatic competence by Japanese learners of English. JALT Journal, 8, 131-155.

U.S. News \& World Report (2013). Regional universities (Midwest). Obtenido de http://colleges.usnews.rankingsandreviews.com/best-colleges/rankings/ regional-universities-midwest

V. G., L. \& Rajan, P. (2012). Non-native student's communication is affected due to the lack of pragmatic competence. English Language Teaching, 5(2), 50-57.

Vellenga, H. E. (2011). Teaching L2 pragmatics: Opportunities for continuing professional development. TESL-EJ, 15(2).

Wedgwood, D. (2012). Pragmatic demands on the form of grammar: Theoretical and methodological limitations on the grammatical code. The Linguistic Review, 29(4), 545-562.

Yamashita, S. O. (1996). Six measures of JSL pragmatics. Honolulu, HI: University of Hawaii. 